\title{
A MOLECULAR DELETION STUDY WITH SOUTHERN HYBRIDIZATION ON TYPICAL PRADER-WILLI SYNDROME (PWS) PATIENTS WITH VARIOUS CHROMOSOME ABNORMALITIES INVOLVING 15q11-12 AND ON AN ATYPICAL PWS PATIENT WITH APPARENTLY NORMAL KARYOTYPE
}

\author{
Tsutomu Kamei, ${ }^{1,}$ * Jun-ichi Hamabe, ${ }^{1}$ Tadashi Matsumoto, ${ }^{1}$ \\ Kyohko Abe, ${ }^{1,7}$ Naoki Harada, ${ }^{1,7}$ Satoshi Ishikiriyama, ${ }^{2}$ \\ Tomoko Hasegawa, ${ }^{3}$ Kiyoshi Miyazaki, ${ }^{4}$ Seiji Mizuno, ${ }^{5}$ \\ Kouji Narahara, ${ }^{6}$ Shigenori Yukizane, ${ }^{8}$ \\ and Norio NIIKAWA ${ }^{1}$
}

\begin{abstract}
${ }^{1}$ Department of Human Genetics, Nagasaki University School of Medicine, Sakamoto-machi 12-4, Nagasaki 852; ${ }^{2}$ Department of Pediatrics, Hokkaido University School of Medicine, Sapporo 060: ${ }^{3}$ Division of Clinical Genetics and Cytogenetics, Shizuoka Children's Hospital, Shizuoka 420; ${ }^{4}$ Department of Pediatrics, Aichi Prefectural Colony Central Hospital, Kasugai 480-03; ${ }^{5}$ Department of Pediatrics, Tokoname City Hospital, Tokoname 479; ${ }^{6}$ Department of Pediatrics, Okayama University Medical School, Okayama 700; ${ }^{7}$ Kyushu Medical Science, Cytogenetics Laboratory, Fukuoka 813; ${ }^{8}$ Department of Pediatrics, Kurume University School of Medicine, Kurume 830, Japan
\end{abstract}

Summary We previously proposed a phenotype-karyotype correlation in the Prader-Willi syndrome (PWS). In order to confirm this hypothesis, we analyzed the genomic DNA of 10 clinically typical PWS patients with various chromosome 15 abnormalities, consisting of four [del(15)(q11.1;q12)], one [del(15)(q11;q13)], one $[-15,+\operatorname{der}(15)($ pter:: ?HSR:: pl1 $\rightarrow$ q11.1:: q12)], one $[\operatorname{mos} 45, \mathrm{X}, \operatorname{del}(15)(\mathrm{q} 11.1 ; \mathrm{q} 12) / 46, \mathrm{X}, \operatorname{del}(15)(\mathrm{q} 11.1 ; \mathrm{q} 12),+\operatorname{mar}]$, two $[\mathrm{t}(15 ; 15)(\mathrm{p} 11.2 ; \mathrm{q} 12)]$ and one $[\operatorname{del}(15)(\mathrm{q} 11.1 ; \mathrm{q} 12),+\mathrm{inv} \operatorname{dup}(15)(\mathrm{q} 11.1 ;$ q11.1)], and that of one atypical PWS patient with an apparently normal karyotype. Densitometric analyses on autoradiographic bands of Southern hybridization using two DNA segments, pML34 and pTD3-21, as probes revealed that all 7 patients with an interstitial deletion of $15 q 11.2$ or $15 q 11.2-$ 12 band had only one copy for each of the probes. In the two patients with an unbalanced translocation $15 \mathrm{q} ; 15 \mathrm{q}$, two copies of each sequence were retained in spite of a visible deletion of band $15 q 11$. This suggests that both of the two sequences may localize in flank of a critical region for the PWS phenotype. The copy number for the two probes was two in the case

Received August 2, 1988; revised version received October 13, 1988; Accepted October 14, 1988

* To whom correspondence should be addressed. 
of inv dup(15), indicating that the patient is not tetrasomic for the sequences corresponding to the two probes. Densitometric analysis of one clinically atypical patient revealed a copy number of two for the two probes. Our results may support both our previous hypothesis and a recently proposed conception that PWS is a contiguous gene syndrome. The results also show that the molecular-genetic analysis is useful for an early and unambiguous diagnosis of PWS. RFLP analyses performed on three patients with $\operatorname{del}(15 q)$ supported the preferential paternal origin of de novo deleted chromosome 15 in PWS.

Key Words Prader-Willi syndrome, chromosome deletion, molecular deletion, contiguous gene syndrome

\section{INTRODUCTION}

Prader-Willi syndrome (PWS) is a multiple congenital anomalies/mental retardation (MCA/MR) syndrome characterized by severe hypotonia and a feeding difficulty in infancy, obesity beginning at early childhood, craniofacial anomalies, short stature, small hands and feet, hypopigmented skin, hypogonadism and mental retardation. At least $50 \%$ or more of the PWS patients have a chromosome- 15 abnormality (Ledbetter et al., 1982; Mattei et al., 1983, 1984; Niikawa and Ishikiriyama, 1985; Labidi and Cassidy, 1986; Butler et al., 1986; Takano et al., 1986). The chromosome abnormalities so far known include: an interstitial deletion [del(15) (q11.1;q12)], a pericentric inversion [inv(15)(p13;q12)], a balanced or unbalanced translocation [t(15q;Dp or other)], an isodicentric or inverted duplication [idic(15) (q11) or inv dup(15)(q11)], and other rearrangements (reviewed by Mattei et al., 1984). In these chromosome abnormalities, a breakpoint in the region 15q11-q12 was always involved, suggesting a localization of the PWS gene(s) within this region. On the other hand, one-third to a half of PWS patients have been reported to have a normal karyotype (Ledbetter et al., 1982, 1987). Thus, there is confusion with regard to the cytogenetic findings on PWS: monosomy (deletion) versus disomy (normal karyotype) versus tetrasomy (inverted duplicated 15q). Mattei et al. (1983) showed that some of the patients with a normal karyotype do not fit the strict criteria for clinical diagnosis of PWS. Niikawa and Ishikiriyama (1985) further found that patients with clinically confirmed PWS always show an interstitial deletion of band $15 \mathrm{q} 11.2$ and proposed a hypothesis of phenotype-karyotype correlation of the disease. However, another confusion has occurred, since recent reports (Schwartz et al., 1985; Reynolds et al., 1987; Kaplan et al., 1987; Magenis et al., 1987) showed that patients with a chromosome $15 \mathrm{q}$ abnormality which was almost identical to that seen in PWS patients lacked some of the features of PWS or had some additional manifestations. Thus, alternative hypothesis was recently proposed by Schmickel (1986) that PWS is a contiguous gene syndrome in which a comprehensive loss of contiguous genes may be involved.

We present here the results of our molecular-deletion analyses on 10 clinically 
typical PWS patients with various chromosome 15 abnormalities and one atypical patient with an apparently normal karyotype, with Southern blot hybridization using two cloned DNA segments specific for $15 \mathrm{q} 11-\mathrm{q} 12$ region as probes. The purposes of our study are to know whether all the cytogenetically visible deletions in PWS patients are confirmed or not at a molecular level, and to confirm the result of cytogenetic studies on the preferential paternal origin of de novo del(15q) by the RFLP analysis.

\section{MATERIALS AND METHODS}

Study subjects. The individuals studied included 10 patients whose phenotypes fit the strict clinical criteria of PWS, one patient who lacks some of the typical features for PWS, and the parents of some of those patients. Six (patient nos. 1-6) of the 10 PWS patients and the atypical patient (patient no. 11) were identical to those reported previously (Niikawa and Ishikiriyama, 1985) (Tables 1 and 2), while the remaining 4 (patient nos. $7-10$ ) were new patients seen directly by us. The clinical manifestations of the 11 patients are shown in Table 1.

Cytogenetic analysis. Every new case was cytogenetically analyzed with highresolution GTG-banding using either the ethidium bromide technique as described previously (Niikawa and Ishikiriyama, 1985) or the BrdU-Actinomycin D method (Rybak et al., 1982).

Southern blot hybridization. Two probes, pTD3-21 and pML34 (kindly provided by Dr. S.A. Latt, Boston), are HindIII subclones of $\lambda$-phage libraries from a flow-sorted inverted duplication chromosome 15 [inv dup(15)(q13)] (Lalande et al., 1985; Donlon et al., 1986). Both pTD3-21 and pML34 (a $2.2 \mathrm{~kb}$ and a $6.4 \mathrm{~kb}$ inserts, respectively) were mapped to the region $15 \mathrm{q} 11.2$ (Donlon et al., 1986). A $0.67 \mathrm{~kb}$ human prealbumin cDNA clone, pPA1 (Mita et al., 1984) assigned to 18q11-q12.3 (Jinno et al., 1986), was used as an internal standard for a comparative analysis of hybridization density. Genomic DNAs of the individuals were extracted by the standard method from their peripheral blood leukocytes or Epstein-Barr virustransformed lymphoblastoid cells. Southern blots of each DNA sample on either nylon or nitrocellulose membranes were hybridized with the triple probes by the standard technique (Maniatis et al., 1982).

Densitometric analysis. The gene doses of both pML34 and pTD3-21 in each individual were determined by taking the average of ratios of their hybridization densities to the PA gene-density obtained from densitometric analyses on several separate hybridizations.

RESULTS

Karyotypes

Of the 4 new PWS patients, one (patient no. 9) showed an interstitial deletion of band $15 \mathrm{q} 11.2[46, \mathrm{XY}, \operatorname{del}(15)(\mathrm{q} 11.1 ; \mathrm{q} 12)]$, one (patient no. 8) had a relatively large 


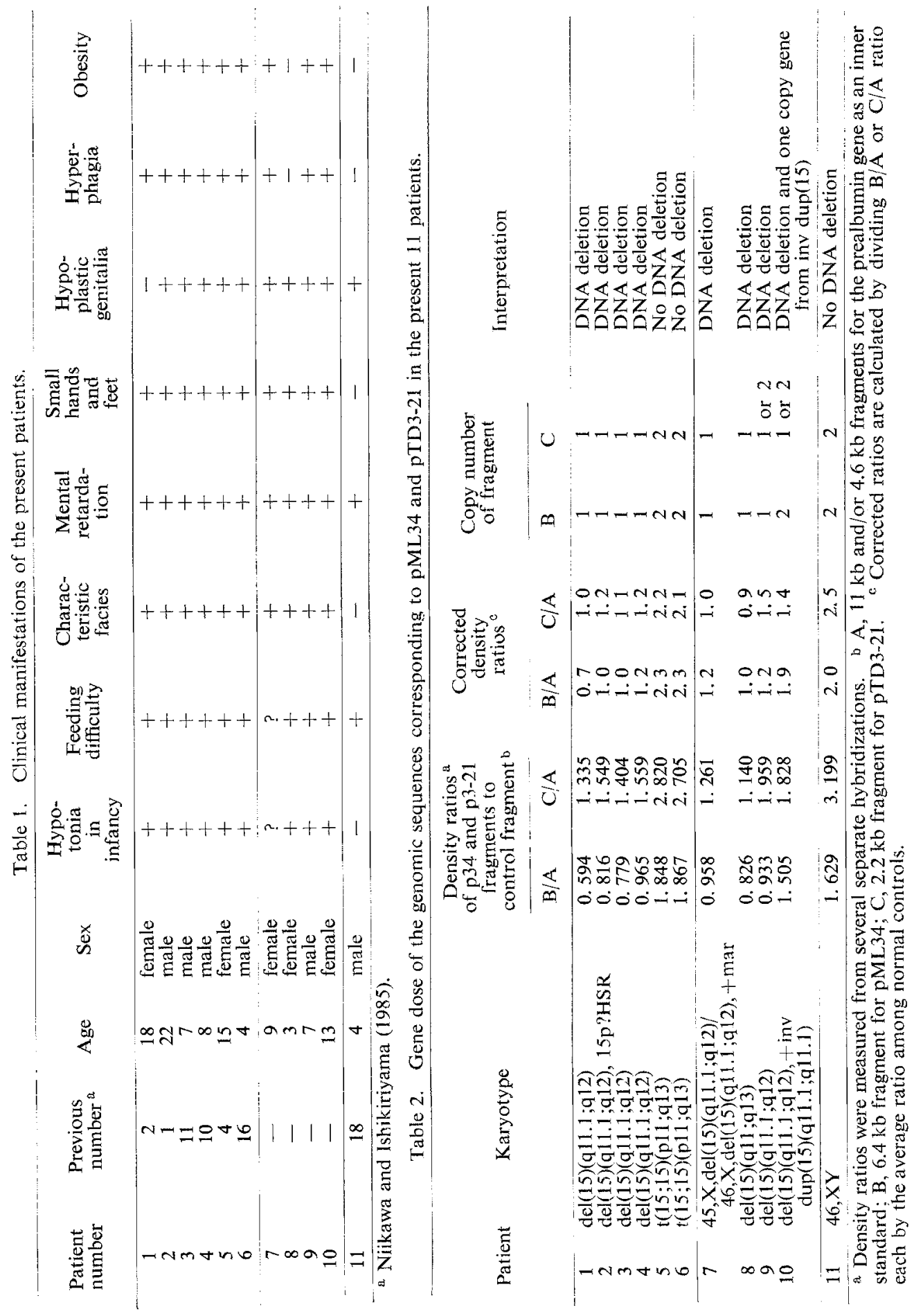


interstitial deletion of $15 \mathrm{q}[46, \mathrm{XX}, \operatorname{del}(15)(\mathrm{q} 11 ; \mathrm{q} 13)]$ (Fig. 1), one (patient no. 10) had a deletion of band $15 \mathrm{q} 11.2$ in one of the homologous members 15 and an additional inverted duplicated chromosome 15 [47, XX, del(15)(q11.1;q12), +inv dup(15) (q11.1;q11.1)], and one (patient no. 7) showed a mosaicism of $[45, X, \operatorname{del}(15)(q 11.1$; q12)/46,X,del(15)(q11.1;q12), + mar] (Fig. 1). No mosaicism was observed in any other patients examined. All parents examined had an apparently normal karyotype.

Gene dose studies

Probes pML34 and pTD3-2I hybridized to a $6.4 \mathrm{~kb}$ and a $2.2 \mathrm{~kb}$ HindIIIfragment, respectively, while the probe pPA1 showed $11 \mathrm{~kb}$ and $4.6 \mathrm{~kb}$ fragments (Jinno et al., 1986). The results of the gene-dose studies on each individual were
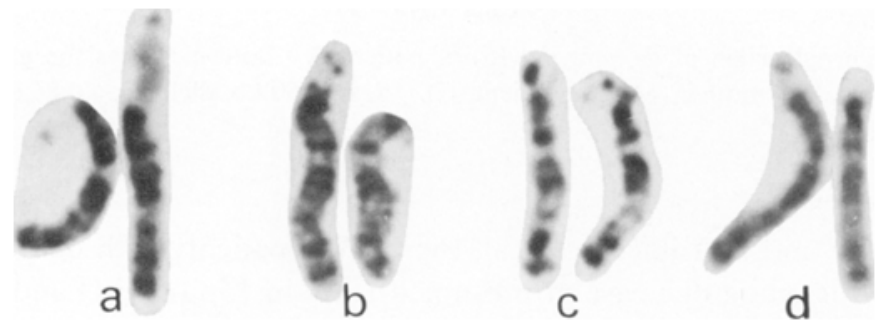

Fig. 1. Chromosome-15 pairs of patients $2($ a) $, 7(b), 8(c)$, and $9($ d) . Right-sided chromosomes are those with $15 \mathrm{q} 11.2$ deletion. Note HSR-like short-arm of chromosome 15 of patient 2 .

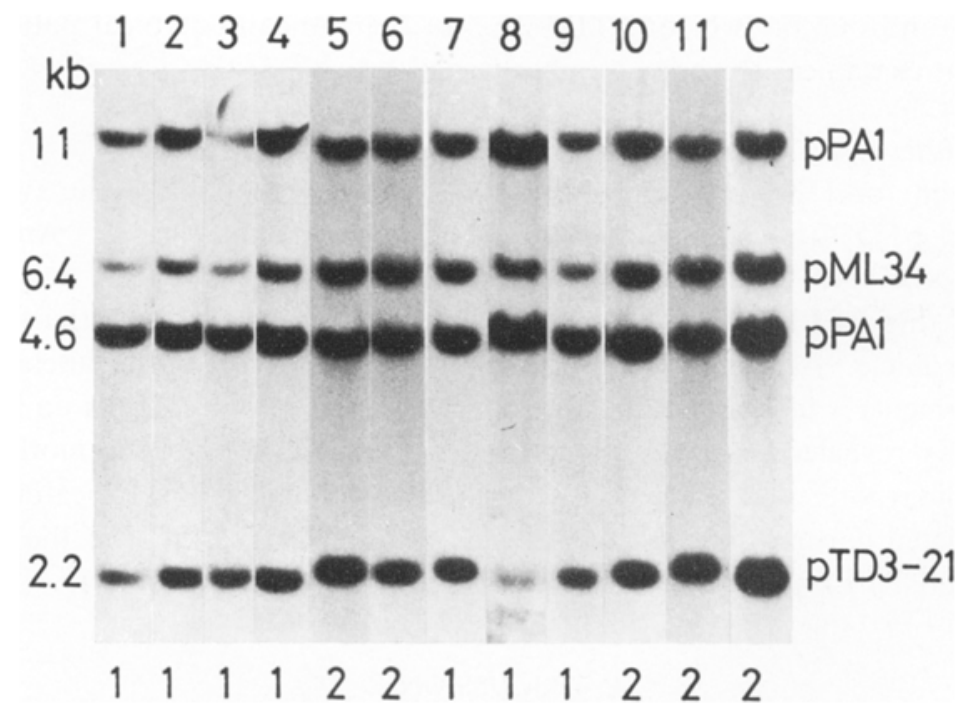

Fig. 2. Autoradiograms of patients with the use of pML34 and pTD3-21 as probes and pPAl as inner control probe. Patient number is shown above the column and copy number of genes (genomic sequences corresponding to pML34 and PTD3-21) is shown at the bottom. 


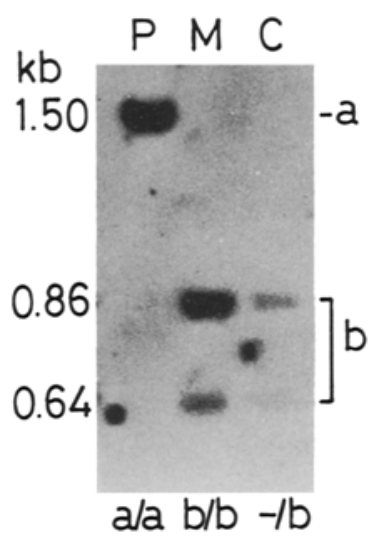

Fig. 3. Paternal origin of de novo del(15) of patient 4. Bottom shows the genotypes of father $(\mathrm{P})$, mother $(\mathrm{M})$ and patient $(\mathrm{C})$. a, an $1.50 \mathrm{~kb}$ allele; $\mathrm{b}$, a $0.86 \mathrm{~kb}+0.64 \mathrm{~kb}$ allele.

shown in Fig. 2 and in Table 2. In all the 7 PWS patients with $\operatorname{del}(15)(\mathrm{q} 11.1 ; \mathrm{q} 12)$ or (q11;q13), including one case of presumed HSR in 15p (Fig. 1) and another case of $45, X$, the hybridization for both pML34 and pTD3-21 showed a one copy density compared with a density of the karyotypically normal control. A density indicating two copies was detected in the two patients with an unbalanced $t(15 q ; 15 q)$. In one PWS patient with a 15q11.2 deletion and an inv dup(15q), the copy number was two for pML34 and one or two for pTD3-21. In the clinically atypical patient and in every parent examined, the copy number was also two.

\section{Parental origin by RFLP analysis}

A search for DNA polymorphism has been carried out for the two probes, pML34 and pTD3-21, using a total of 13 restriction endonucleases. An RFLP of $1.5 \mathrm{~kb} / 0.86+0.64 \mathrm{~kb}$ was detected only with a combination of AluI and pTD3-21. A study of this RFLP among ten normal individuals revealed that the frequencies for a major allele "A1" (a $0.86 \mathrm{~kb}+0.64 \mathrm{~kb}$ fragment) and a minor allele "A2" (an $1.5 \mathrm{~kb}$ fragment) is 0.80 and 0.20 , respectively. Segregation analysis on this RFLP in one family revealed that the father is an A1/A1 homozygote, the mother was an A2/A2 homozygote, and the patient was an A2/- hemizygote, indicating that the de novo deleted chromosome 15 was of paternal origin (Fig. 3). In the other two patients, AluI-RFLP was uninformative for the deleted chromosome 15 .

\section{DISCUSSION}

Cytogenetic analyses on four new PWS patients in our series confirmed our previous conclusion of a phenotype-karyotype correlation in the syndrome (Niikawa and Ishikiriyama, 1985). The results of the present molecular study also confirmed 
the previous findings by Donlon et al. (1986) and by Latt et al. (1987), except for the frequency of the molecular deletion. All our patients with an interstitial deletion of 15q11.2-q13 had a molecular deletion without any exceptions, while with the same probes, Donlon et al. (1986) found a DNA deletion in only one of their two PWS patients with the similar chromosome deletion as seen in our patients. A further study using four other chromosome-15 DNA clones (pIR4-3, pIR10-1, p189-1 and p190-2) as probes by the same group of investigators (Latt et al., 1987) revealed a deletion at a molecular level in three of six PWS patients studied and probably in a PWS-like patient. The discrepancy of molecular deletion frequency between our and their studies is most likely due to a chance in collectioning patients in our study whose chromosome deletions would have been uniform.

Against all expectations, both of the patients with an unbalanced translocations $15 q ; 15 q$ were shown to retain the two DNA sequences in a double dose. This unexpected finding is hard to be explained by an assumption that the both of pML34 and pTD3-21 would be localized in flank to but apart from putative gene(s) responsible for the PWS phenotype, since the range of the deletion in each translocation is from 15 pter to $15 q 12$, which is wider than that in the interstitial deletion cases. Therefore, more complex rearrangements of DNA might have occurred in the genome of these translocation patients. Alternatively, although we could not have observed any abnormality other than the translocation in these patients (Niikawa and Ishikiriyama, 1985), the critical DNA segments might have inserted into another chromosome.

A two copy density of the sequence for each probe was detected in the genome of the patient with an interstitial deletion of band 15q11.2 accompanied with an additional inverted duplicated chromosome 15. This finding indicates that the patient is not tetrasomic at least for both the pML34 and pTD3-21 sequences, and is consistent with the result of the study on a cell line containing a small inv dup(15) by Donlon et al. (1986). One copy of each gene in our patient must have been derived from his one intact chromosome 15 , while the other one copy may have come either from a deleted chromosome 15 or from an inverted duplicated chromosome.

Although we could not detect any cytogenetic and molecular deletions in an atypical patient, our finding may not universally be applicable to other atypical PWS patients, since the probes we used are the sequences probably flanking to the region responsible for the PWS phenotypes (Donlon et al., 1986). More studies on such atypical patients are necessary using the same or the other probes as isolated by Latt et al. (1987).

The heterogeneity of the molecular changes observed in the present study on PWS patients may reflect instability around the $15 q 11$ region, i.e., a rearrangement hot-spot. This unstable region is physically closely linked to the critical region for the common phenotype of PWS. This "hot-spot" hypothesis first proposed by Riccardi (1981) and then by Mattei et al. (1984) may be compatible with the result of a study by Donlon et al. (1986) showing that among a total genomic library, two inserts, IR-4 and IR-10, localized at the 15 q 11.2 region contain an inverted repeat 
DNA sequence which is belonging to the $A / u$ family. Furthermore, chromosome rearrangements involving 15 q11-q13 have been observed in patients with malformation syndromes or in those with well-known syndrome-like disorders other than PWS. These disorders include Cohen-like syndrome (Fuhrmann-Rieger et al., 1984), Williams syndrome (Fryns et al., 1982; Kaplan et al., 1987), Angelman syndrome (Kaplan et al., 1987), and many other disorders with dysmorphic manifestations (reviewed by Mattei et al., 1984). An one copy density per genome for both pML34 and pTD3-21 was confirmed in one Angelman syndrome patient in our preliminary study (described elsewhere).

The chromosome abnormality of one of our PWS patients (patient no. 2) merits comment. The detailed clinical manifestations and family data were described previously (Kato et al., 1965; Niikawa and Ishikiriyama, 1985). In short, he, his father and several other family members have an unusual chromosome 15 with an extremely long short-arm that appears a homogeneously staining region (HSR)-like structure by GTG- and QFQ-bandings (Fig. 1). A loss of genetic material in 15q11.2 region. in this unusual chromosome 15 was detected only in the patient at both microscopic and molecular levels, and the HS-like region itself gives no phenotypic effects on any individuals who carry it. It is of great interest to know the molecular structure of this HS-like region. If we assume that this contains a highly repeated DNA sequence resulted from a pericentric inversion involving the $15 \mathrm{q} 11$ region and subsequent tandem repeats, it would reflect the instability of the region. Then, the loss of the sequences, pML34 and pTD3-21, in the patient could also be explained by the recombinant aneusomy mechanism as a result of a loop formation around the centromere. Thus, a further molecular study is necessary using a highly repetitive cloned DNA sequence as a probe, as isolated by Donlon et al. (1986).

The findings in our study together with the previous molecular, cytogenetic and clinical studies provide us the following conclusions: (1) The gene(s) located in $15 q 11.2$ region play an important role in the cause of PWS; $(2)$ there is not only genetic but also phenotypical heterogeneity in the rearrangements involving the $15 \mathrm{q} 11$ q12 region; (3) this heterogeneity may be compatible with a conception, "the contiguous gene syndrome," induced recently by Schmickel (1986). If we follow this conception, each DNA segment (gene) in the region $15 \mathrm{q} 11.2$ would be responsible for each component of the common phenotype of PWS. In addition, if we extend this conception into a syndrome scale, the gene(s) for PWS would flank or overlap to a segment for Angelman syndrome, and possibly those for Williams and Cohen syndromes. Among the heterogeneous classes of deletion, a comprehensive loss of a critical segment which may contain putative gene(s) would lead to a syndrome, while a loss of a larger or a smaller segment would result in variation of phenotypes.

Our results on the parental origin of the de novo deleted chromosomes 15 in PWS patients traced with molecular probes are consistent with the preferential paternal origin of a deleted chromosome in PWS patients that was ascertained in previous cytogenetic studies (Butler and Palmer, 1983; Hasegawa et al., 1984; Niikawa and Ishikiriyama, 1985). This preferential paternal origin is also consistent 
with the data in other de novo non-Robertsonian structural rearrangements (Chamberlin and Magenis, 1980).

A few PWS patients are familial (Hasegawa et al., 1984; Lubinsky et al., 1987; Cassidy, 1987). Prenatal diagnosis in a pregnancy following the birth of a PWS child have been performed cytogenetically (Emanuel et al., 1983; Smith, 1986), but no recurrence has been found in the pregnancies studied (Schinzel, 1986). It is often difficult to obtain convincing prometaphase chromosomes from cultured amniotic cells. Thus, molecular-genetic analysis may be useful for prenatal diagnosis, especially in familial cases. Neonatal diagnosis of PWS is more important clinically to prevent patients early from obesity and its subsequent diabetes. However, the clinical diagnosis in early infancy is often difficult, because the time of onset of obesity is usually in around childhood. Although neonatal diagnosis of PWS by chromosome analysis was recently succeeded (Greenberg et al., 1987), a molecular genetic analysis as described here is also applicable to the diagnosis of presumed newborn patients, especially of patients with a normal karyotype.

Acknowledgment We gratefully acknowledge Professor Samuel A. Latt, Harvard Medical School for providing the molecular probes and Dr. Martin C. Wapenaar, University Leiden, for his critical reading of this manuscript. This study was supported by a Grant-in-Aid for Scientific Research (no. 63480472) from the Ministry of Education, Science and Culture of Japan, and also supported in part by Ministry of Health and Welfare of Japan.

\section{REFERENCES}

Butler, M.G. and Palmer, C.G. 1983. Parental origin of chromosome 15 deletion in Prader-Willi syndrome. Lancet i: 1285-1286.

Butler, M.G., Meaney, F.J., and Palmer, C.G. 1986. Clinical and cytogenetic survey of 39 individuals with Prader-Labhart-Willi syndrome. Am. J. Med. Genet. 23: 793-809.

Cassidy, S.B. 1987. Recurrence risk in Prader-Willi syndrome. Am. J. Med. Genet. 28: 59-60.

Chamberlin, J. and Magenis, R.E. 1980. Parental origin of de novo chromosome rearrangements. Hum. Genet. 53: 343-347.

Donlon, T.A., Lalande, M., Wyman, A., Bruns, G., and Latt, S.A. $1986 . \quad$ Isolation of molecular probes associated with the chromosome 15 instability in the Prader-Willi syndrome. Proc. Natl. Acad. Sci. USA 83: 4408-4412.

Emanuel, M., Mak, E., Gryfe, A., and Wyatt, P. 1983. Prader-Willi syndrome and prenatal diagnosis. Lancet ii: 1363.

Fryns, J.P., van den Hauwaert, L.G., DuMoulin, M., and van den Berghe, H. 1982. The elfin face syndrome and the short arm of chromosome 15. Ann. Génét. 25: 181-182.

Fuhrmann-Rieger, A., Kohler, A., and Fuhrmann, W. 1984. Duplication or insertion in 15q11-13 associated with mental retardation-short stature and obesity-Prader-Willi or Cohen syndrome? Clin. Genet. 25: 347-352.

Greenberg, F., Elder, F.F.B., and Ledbetter, D.H. 1987. Neonatal diagnosis of Prader-Willi syndrome and its implications. Am. J. Med. Genet. 28: 845-856.

Hasegawa, T., Hara, M., Ando, M., Osawa, M., Fukuyama, Y., Takahashi, M., and Yamada, K. 1984. Cytogenetic studies of familial Prader-Willi syndrome. Hum. Genet. 65: 325-330.

Jinno, Y., Matsumoto, T., Kamei, T., Kondoh, T., Maeda, S., Araki, S., Shimada, K., and Niikawa, N. 1986. Localization of the human prealbumin gene to $18 \mathrm{p} 11.1-\mathrm{q} 12.3$ by gene dose effect study of Southern blot hybridization. Jpn. J. Human Genet. 31: 243-248. 
Kaplan, L.C., Wharton, R., Elias, E., Mandell, F., Donlon, T., and Latt, S.A. 1987. Clinical heterogeneity associated with deletions in the long arm of chromosome 15: Report of 3 new cases and their possible genetic significance. Am. $J$. Med. Genet. 28: 45-53.

Kato, H., Omura, T., Tabata, S., and Makino, S. 1965. A preliminary note on an aberrant autosome (Group 13-15) in a male and in his father. Proc. Jpn. Acad. 41: 477-481.

Labidi, F. and Cassidy, S.B. 1986. A blind prometaphase study of Prader-Willi syndrome: Frequency and consistency in interpretation of del 15q. Am.J. Hum. Genet. 39: 452-460.

Lalande, M., Schreck, R.R., Hoffman, R., and Latt, S.A. 1985. Identification of inverted duplicated \#15 chromosomes using bivariate flow cytometric analysis. Cytometry 6: 1-6.

Latt, S.A., Tantravahi, U., Neve, R., Nicholls, R., Ringer, S., Stroh, H., Fuller, R., Donlon, T., Kaplan, L., and Wharton, R. 1987. New and better molecular probes for characterizing and ultimately understanding the consequences of chromosome 15 deletions in the Prader-Willi syndrome. Am. J. Med. Genet. 28: 917-918.

Ledbetter, D.H., Mascarello, J.T., Riccardi, V.M., Harper, V.D., Airhart, S.D., and Strobel, R.J. 1982. Chromosome 15 abnormalities and the Prader-Willi syndrome: A follow-up report of 40 cases. Am. J. Hum. Genet. 34: 278-285.

Ledbetter, D.H., Greenberg, F., Holm, V.A., and Cassidy, S.B. 1987 . Conference report: Second Annual Prader-Willi Syndrome Scientific Conference. Am. J. Med. Genet. 28: 779-790.

Lubinsky, M., Zellweger, H., Greenswag, L., Larson, G., Hansmann, I, and Ledbetter, D. 1987. Familial Pradew-Willi syndrome with apparently normal chromosomes. Am. J. Med. Genet. 28: $37-43$.

Magenis, R.E., Brown, M.G., Lacy, D.A., Budden, S., and LaFranchi, S. 1987. Is Angelman syndrome an alternate result of $\operatorname{del}(15)(\mathrm{q} 11 \mathrm{q} 13)$ ? Am. J. Med. Genet. 28: 829-838.

Maniatis, T., Fritsch, E.F., and Sambrook, J. 1982. In Molecular Cloning: A Laboratory Manual, Cold Spring Harbor, New York, pp. 150-172, 382-389.

Mattei, J.F., Mattei, M.G., and Giraud, F. 1983. Prader-Willi syndrome and chromosome 15. Hum. Genet. 64: 356-362.

Mattei, M.G., Souiah, M., and Mattei, J.F. 1984. Chromosome 15 anomalies and the PraderWilli syndrome: Cytogenetic analysis. Hum. Genet, 66: 313-334.

Mita, S., Maeda, S., Shimada, K., and Araki, S. 1984. Cloning and sequence analysis of cDNA for human prealbumin. Biochem. Biphys. Res. Commun. 124: 558-564.

Niikawa, N. and Ishikiriyama, S. 1985. Clinical and cytogenetic studies of the Prader-Willi syndrome: Evidence of phenotype-karyotype correlation. Hum. Genet. 69: 22-27.

Reynolds, J.F., Daniel, A., and FitzGerald, J. 1987. Brief clinical report: Atypical phenotype associated with deletion (15)(pter-q11::q13-qter). Am. J. Med. Genet. 28: 55-58.

Riccardi, V.M. 1981. The Prader-Willi syndrome (PWS) as a "Transposable Elements" disorder. Am. J. Hum. Genet. 33: 117A only.

Rybak, J., Tharapel, A., Robinett, S., Garcia, M., Mankinen, C., and Freeman, H. 1982. A simple reproducible method for prometaphase chromosome analysis. Hum. Genet. 60: 328-333.

Schinzel, A. 1986. Approaches to the prenatal diagnosis of the Prader-Willi syndrome. Hum. Genet. 74: 327.

Schmickel, R.D. 1986. Contiguous gene syndromes: A component of recognizable syndromes. J, Pediatr. 109: 213-241.

Schwartz, S., Max, S.R., Panny, S.R., and Cohen, M.M. 1985. Deletions of proximal 15q and non-classical Prader-Willi syndrome phenotypes. Am. J. Med. Genet. 20: 255-263.

Smith, A. 1986. Prenatal diagnosis and the Prader-Willi syndrome. Hum. Genet. 72: 278.

Takano, T., Nakagome, Y., Nagafuchi, S., Tanaka, F., Nakamura, Y., Nagano, T., Tanae, A., and Hibi, I. 1986. High-resolution cytogenetic studies in patients with Prader-Willi syndrome. Clin. Genet. 30: 241-248. 